\title{
Efeito Anti-Tumoral dos Bisfosfonatos: uma Nova Perspectiva Terapêutica
}

\author{
Anti-Tumor Effect of Bisphosphonates: a New Therapeutic Perspective
}

Déborah V. Vasconcellos ', Maria Eugenia L. Duarte ${ }^{2}$, Raquel C. Maia ${ }^{3}$

\begin{abstract}
Resumo
O s bisfosfonatos (BFs) são potentes inibidores da reabsorção óssea mediada por osteoclastos. Essas drogas são efetivas na redução do cálcio sérico em pacientes com hipercalcemia maligna, assim como também no tratamento da dor óssea, osteoporose e metástases ósseas.

Diversos estudos demonstram que os BFs possuem efeito em outras células além dos osteoclastos. Em células tumorais podem agir induzindo a apoptose, inibindo a proliferação celular, inibindo a adesão e a invasividade celular ou as metástases ósseas. 0 mecanismo molecular subjacente a estes efeitos parece ser a inibição de enzimas da via do mevalonato, o que leva a um impedimento da prenilação de GT Pases como Ras e R ho, importantes para a manutenção da integridade do citoesqueleto e tráfego de vesículas nas células.

As evidências dos recentes estudos laboratoriais e clínicos sugerem que os BFs têm um papel importante como tratamento suplementar e possivelmente complementar na terapia do câncer. Um entendimento mais profundo e completo sobre o efeito anti-tumoral destas drogas pode sugerir possibilidades terapêuticas novas e seletivas.

Palavras-chave: efeito anti-tumoral; bisfosfonatos; metástases; apoptose.
\end{abstract}

\begin{abstract}
Bisphosphonates are potent osteoclast-mediated bone reabsorption inhibitors. These drugs are effective in reducing serum calcium levels in patients with malignant hypercalcaemia, as well as in bone pain, osteoporosis and bone metastasis treatment.

Several studies have demonstrated that bisphosphonates are effective in other cell types than osteoclasts. In tumour cells they can act inducing apoptosis, inhibiting cell proliferation, inhibiting cell adhesion and invasion or bone metastasis. The underlying molecular mechanism to these effects seems to be the inhibition of mevalonate pathway enzymes, which leads to a prenilation impairment of GT Pases like Ras and R ho, important in maintaining celular cytoskeleton integrity and vesicles trafficking.

Evidences from recent laboratorial and clinical studies suggest that bisphosphonates have an important role as a supplemental, and possibly as complementary treatment in cancer therapy. A more complete understanding concerning the anti-tumor effect of these drugs may suggest new and selective therapeutical possibilities.
\end{abstract}

Key words: anti-tumor effect; bisphosphonates; metastasis; apoptosis.

\footnotetext{
1. M estranda do Laboratório de H ematologia Celular e M olecular do H ospital do Câncer I (HC-I) / Instituto N acional de Câncer (IN CA) e do Programa de Pós-graduação em Ciências M orfológicas do Instituto de Ciências Biomédicas da U niversidade Federal do Rio de Janeiro (UFRJ) Rio de Janeiro - Brasil.

2 - Professora do D epartamento de H istologia e Embriologia da UFRJ - Rio de Janeiro - Brasil

3. M édica H ematologista, PhD. Chefe do Laboratório de H ematologia Celular e M olecular do H ospital do Câncer I / IN CA - Rio de Janeiro Brasil.

Enviar correspondência para: Raquel C. M aia - Laboratório de H ematologia Celular e M olecular - H ospital do Câncer I - Instituto N acional de Câncer - Praça da Cruz Vermelha, 23/70 andar - Centro - Rio de Janeiro - Brasil - email: rcmaia@inca.gov.br
} 


\section{BISFOSFONATOS}

O s bisfosfonatos (BFs) são análogos do pirofosfato endógeno, no qual um átomo de carbono substitui o átomo central de oxigênio. Propriedades do pirofosfato indicavam que este poderia ser explorado em doenças de calcificação ectópica ou de aumento da reabsorção ósseal. No entanto, a incapacidade de o pirofosfato agir quando administrado oralmente, inibindo a reabsorção óssea, propiciou o desenvolvimento de análogos com ações similares, porém mais resistentes à ação enzimática, como é o caso dos $B F s^{2}{ }^{2}$ In vivo, os BFs se ligam fortemente à hidroxiapatita da superfície óssea e atuam preferencialmente em sítios de grande formação e reabsorção ósseas. ${ }^{1}$

Todos os BFs possuem propriedades fisico-químicas e farmacocinéticas similares. Geralmente esses compostos são fracamente adsorvidos a partir do trato gastrointestinal por serem pouco lipofílicos. Os BFs circulantes desaparecem rapidamente do plasma e aproximadamente a metade é capturada pelo osso, sendo o restante excretado sem modificações pelos rins. Essa classe de drogas não é metabolizada pelo fígado., ${ }^{3,4}$

\section{INDICAÇÕES CLÍNICAS PARA O USO DOS BISFOSFONATOS}

Após mais de três décadas de pesquisas e desenvolvimento, os BF s tornaram-se indispensáveis no tratamento de doenças ósseas, tanto benignas quanto malignas. ${ }^{5,6}$ Essas drogas são potentes inibidores da reabsorção óssea mediada por osteoclastos ${ }^{7}$ e são efetivas na redução das concentrações de cálcio no soro de pacientes com hipercalcemia maligna. ${ }^{8,9}$ Além disso, eles são uma nova modalidade de tratamento para metástases ósseas, já que reduzem a quantidade e a taxa de complicações esqueléticas no mieloma múltiplo (M M) e no câncer de mama avançado ${ }^{10,11}$, e também aliviam a dor óssea causada por metástases de vários tumores sólidos, com conseqüente aumento da qualidade de vida do paciente (Q uadro 1). ${ }^{12,13}$

\section{MECANISMOS DE AÇÃO DOS BISFOSFONATOS}

Os mecanismos pelos quais os BFs atuam na reabsorção óssea em nível celular envolvem provavelmentea inibição da formação e/ ou recrutamento de osteoclastos a partir de células precursoras imaturas, ${ }^{7,14}$ inibição da ativação de osteoclastos, inibição da atividade de osteoclastos maduros e/ou indução de apoptose. $15,16,17$

A inibição da formação de osteoclastos foi demonstrada com vários BFs em sistemas experimentais in vitro, nos quais osteoclastos foram gerados a partir da medula óssea. ${ }^{14}$ Seu papel in vivo é evidenciado pela cinética da inibição da reabsorção e a redução do número de osteoclastos após tratamentos prolongados. ${ }^{17}$

Atualmente é aceito que muitos fatores que estimulam a reabsorção óssea mediada por osteoclastos, tais como paratormônio, prostaglandina $E$, interleucina-1 efator de necrosetumoral a (TN Fa), agem indiretamente via osteoblastos. Existem evidências de que os osteoblastos produzem fatores que estimulam a atividade de osteoclastos maduros. ${ }^{17} 0$ wens et al. demonstraram que 0 alendronato (um tipo de BF) não afetava o número de osteoclastos de ratos in vitro, porém reduzia sua atividade em determinada concentração na presença de osteoblastos. ${ }^{18} \mathrm{Na}$ ausência de osteoblastos, a concentração necessária para a redução da atividade dos osteoclastos era maior, sugerindo que o mecanismo de ação dos BFs também se dá via inibição da ativação de osteoclastos por osteoblastos. ${ }^{17}$

A inibição direta da atividade dos osteoclastos é sugerida por observações in vivo do desaparecimento da borda ondulada, que é a membrana convoluta dos osteoclastos associada a sua atividade. ${ }^{19,20}$ Essa membrana parece ser o sítio do tráfego bidirecional: enzimas lisossomais e prótons são liberados no hemivacúolo de reabsorção, que age como um lisossomo gigante, enquanto o cálcio e os produtos de degradação da matriz são internalizados e transportados via transcitose para a membrana baso-lateral da célula. ${ }^{21,22} 0$ steoclastos

Quadro 1. Indicações clínicas para o uso dos bisfosfonatos

- Tratamento da hipercalcemia induzida por tumor

- Tratamento da dor óssea;

- Redução de complicações esqueléticas em pacientes com metástases ósseas ou mieloma múltiplo;

- Tratamento da doença de Paget óssea;

- Tratamento da osteoporose pós-menopausa;

- Prevenção da perda óssea causada por tratamento com glicocorticóides;

- Aumento da densidade óssea na fibrodisplasia;

- Alívio dos sintomas da osteogênese imperfecta. 
examinados em microscópio eletrônico após administração de alendronato não possuíam a membrana convoluta e pareciam quiescentes. ${ }^{20}$ Essa observação é uma forte indicação da inativação de osteoclastos por BFs in vivo. ${ }^{17}$

A apoptose, ou morte celular programada, foi descrita em osteoclastos há muitos anos e talvez seja a via normal de morte dessas células. ${ }^{17} 0$ tempo de vida dos osteoclastos, estimado a partir de estudos histológicos, é de duas a quatro semanas in vivo e de no máximo duas semanas in vitro. Já foi descrito que os BFs aumentam a apoptose de osteoclastos, tanto in vivo quanto in vitro. ${ }^{15}$

Os BFs podem ser divididos em dois subgrupos, dependendo da presença ou não de nitrogênio. Pesquisas recentes sugerem que os primeiros BFs usados clinicamente e não-aminados (etidronato e clodronato) são metabolizados em análogos citotóxicos de ATP ${ }^{23}$, sendo estes os responsáveis pela inibição da atividade dos osteoclastos. Por outro lado, os BFs aminados, mais novos e potentes - pamidronato, ibandronato, alendronato e zoledronato -, induzem apoptose nos osteoclastos, inibindo enzimas da via do mevalonato e prevenindo a prenilação (principalmente a geranilgeranilação) de pequenas proténas que se ligam a GTP, que são essenciais para o tráfego de vesículas e manutenção da integridade do citoesqueleto. $6,15,24,25,26,27,28$
A geranilgeranilação é a ligação de um lipídio de 20 carbonos (geranilgeranil) a certas proteínas, incluindo proteínas-G regulatórias chaves, tais como Rac, Rho, C dc-42, e vários membros da família Rab. A geranilgeranilação é necessária para ancorar essas proteínas na membrana plasmática ou em membranas intracelulares. A ausência desse processo resulta em um bloqueio ou mudança em eventos sinalizadores que em última instância levam à indução das caspases e à apoptose (Figura 1). 25,27,29

Foi demonstrado que a apoptose induzida por BFs em osteoclastos envolve mudanças morfológicas características, perda de potencial de membrana da mitocôndria e ativação de caspases, sendo que a caspase 3 parece ser a principal caspase efetora ativada no processo. ${ }^{30} \mathrm{Em}$ um outro trabalho, todos os BFs utilizados induziram a formação de núcleo picnótico dependente de caspase e a clivagem da $M$ stl kinase para formar a espécie ativa de $34 \mathrm{kD}$ a, o que sugere que os BFs agem diretamente nos osteoclastos induzindo apoptose. ${ }^{27}$ Porém, em trabalho publicado por Benford et al., apenas os BFs aminados causaram um aumento na atividade de proteases "caspase-3-like" em macrófagos J774, não sendo observada nos BFs nãoaminados nem em concentrações que sabidamente reduzem a viabilidade ou inibem a proliferação celular. ${ }^{25}$

Figura 1. Mecanismo de ação dos bisfosfonatos

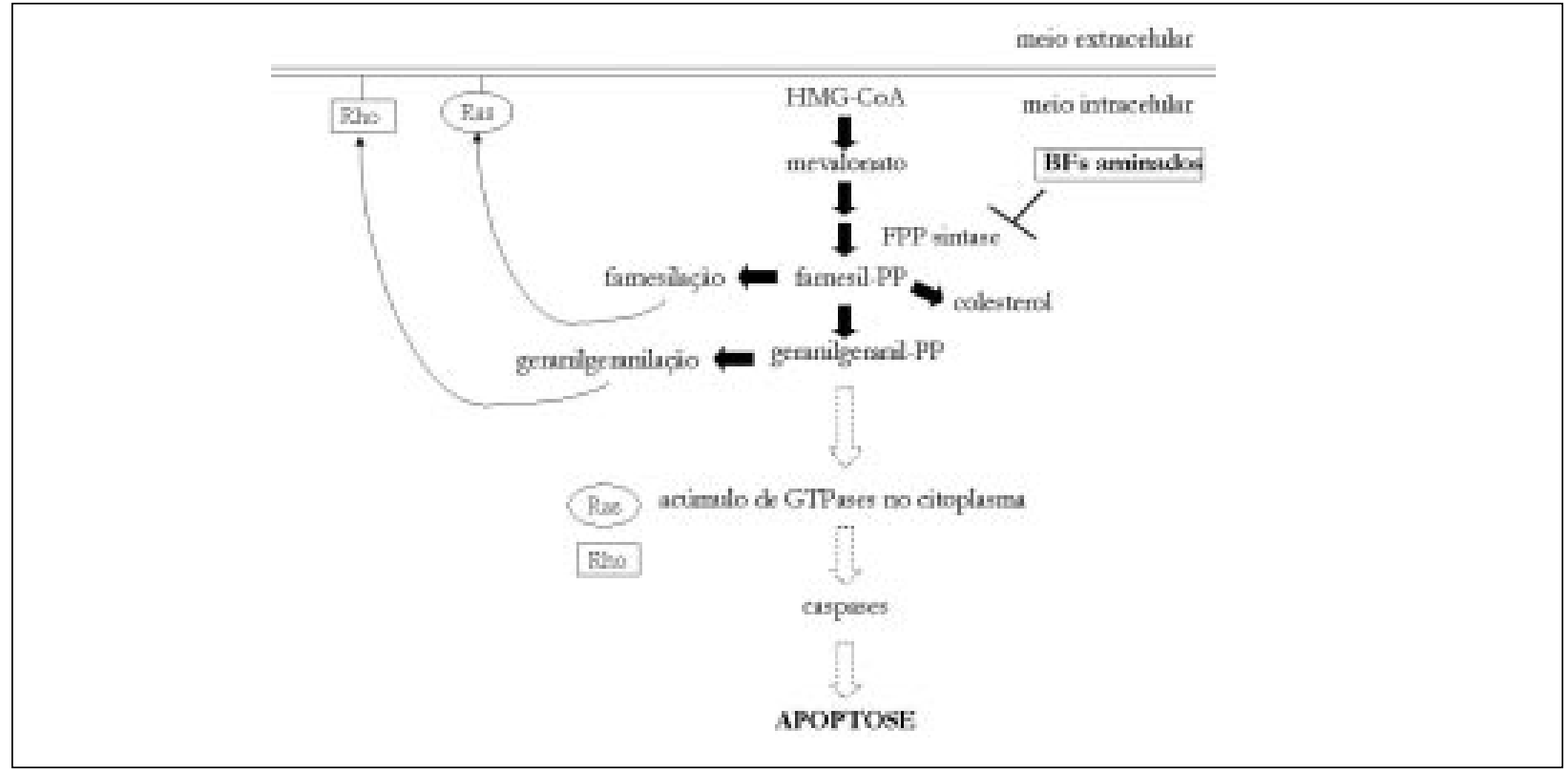

O s BFs aminados induzem a apoptose inibindo a farnesil pirofosfato sintase (FPP sintase) e prevenindo a prenilação de pequenas GT Pases, que são essenciais para o tráfego de vesículas e manutenção da integridade do citoesqueleto. A prenilação é necessária para ancorar essas proteínas na membrana plasmática ou em membranas intracelulares. A ausência desse processo resulta em um bloqueio ou mudança em eventos sinalizadores que em última instância levam à indução das caspases e à apoptose. 
MECANISMOS DE AÇÃO DOS BISFOSFONATOS NOS TUMORES

Várias pesquisas demonstraram que os BFs também possuem efeito em outras células além dos osteoclastos, incluindo células tumorais. Já existe uma quantidade considerável de dados in vitro demonstrando que os BFs têm ação citostática, induzem apoptose e inibem a adesão ea invasividade de células tumorais (Tabela 1), interferem no processo metastático (Tabela 2), têm efeito na secreção de fatores de crescimento e citocinas e inibem a angiogênese tumoral (Tabela 3$)^{6}{ }^{6}$

Embora em menor escala, evidências in vivo demonstrando a ação dos BFs em tumores estão começando a se acumular. ${ }^{6}$

\section{Inibição da proliferação celular e indução de apoptose}

Trabalhos recentes indicam que BFs podem ter um efeito anti-proliferativo direto e induzir apoptose tanto em linhagens de células de $M M$ humano ${ }^{31}$ quanto em plasmócitos de pacientes com M M. . $^{32} \mathrm{~A}$ adição de geranilgeraniol e farnesol, dois intermediários da via do mevalonato, previne a apoptose induzida pelos BFs e reverte parcialmente a interrupção do ciclo celular, ${ }^{33}$ sugerindo que seu mecanismo de ação em células de
M M seja o mesmo que nos osteoclastos. Células da linhagem de mieloma IM -9 transfectadas com um vetor expressando a proteína anti-apoptótica Bcl-2 mostraramse completamente protegidas contra a apoptose, demonstrando viabilidade normal e DNA intacto. ${ }^{32}$

G ordon et al. observaram que uma única infusão de pamidronato em 16 pacientes com M M causou um aumento importante na apoptose de plasmócitos in vivo em 10 pacientes e um pequeno aumento em 4 deles. ${ }^{34}$ O s BF s pamidronato e zoledronato também induziram apoptose em plasmócitos humanos in vitro, provavelmente causada pela inibição da via do mevalonato e perda de pequenas $\mathrm{G} T$ Pases, já que mesmo concentrações baixas de zoledronato causaram acúmulo de Rap1A não-preniladas em culturas de células mononucleares da medula óssea. ${ }^{34}$

Em trabalho publicado por Tassone et al. foi observado que a combinação do BF zoledronato com dexametasona, esta última normalmente utilizada no tratamento de pacientes com $M M$, possui efeito sinérgico em linhagens celulares de mieloma humano in vitro, 0 que leva a uma interessante perspectiva sobre essa combinação terapêutica in vivo. ${ }^{35}$

Em trabalho recente realizado por $\mathrm{N}$ ishida et al. foi

Tabela 1. Trabalhos pré-clínicos sobre a ação anti-tumoral dos bisfosfonatos em linhagens tumorais

\begin{tabular}{|c|c|c|}
\hline Autor & Linhagem & Propriedade anti-tumoral \\
\hline Van der Pluijm et al., 1996 & câncer de mama & inibição da adesão celular \\
\hline Boissier et al., 1997 & $\begin{array}{l}\text { câncer de mama e de } \\
\text { próstata }\end{array}$ & inibição da adesão celular \\
\hline Shipman et al., 1997, 1998 & mieloma múltiplo & indução de apoptose \\
\hline Stearns, 1998 & câncer de próstata & inibição da invasividade celular \\
\hline Aparicio et al., 1998 & mieloma múltiplo & indução de apoptose \\
\hline Tassone et al., 2000 & mieloma múltiplo & indução de apoptose \\
\hline Fromigue et al., 2000 & câncer de mama & indução de apoptose \\
\hline Boissier et al., 2000 & $\begin{array}{l}\text { câncer de mama e de } \\
\text { próstata }\end{array}$ & inibição da invasividade celular \\
\hline Senaratne et al., 2000, 2002 & câncer de mama & indução de apoptose \\
\hline Jagdev et al., 2001 & câncer de mama & indução de apoptose \\
\hline Sonnemann et al., 2001 & osteossarcoma & inibição da proliferação \\
\hline Riebeling et al., 2002 & melanoma & indução de apoptose \\
\hline Virtanen et al., 2002 & câncer de próstata & $\begin{array}{c}\text { inibição da adesão, invasividade e } \\
\text { migração celular }\end{array}$ \\
\hline
\end{tabular}


Tabela 2. Trabalhos pré-clínicos sobre a ação dos bisfosfonatos em modelos animais

\begin{tabular}{|c|c|c|}
\hline Autor & Modelo animal & Propriedade anti -tumoral \\
\hline Wingen et al., 1986 & $\begin{array}{c}\text { Ratos SD + linhagem de } \\
\text { carcinossarcoma de Walker } 256\end{array}$ & Redução da osteólise tumoral \\
\hline $\begin{array}{l}\text { Krempien et al., } \\
1988\end{array}$ & $\begin{array}{c}\text { Ratos + linhagem de } \\
\text { carcinossarcoma de Walker } 256\end{array}$ & $\begin{array}{c}\text { Inibição da destruição óssea e da } \\
\text { hipercalcemia }\end{array}$ \\
\hline Hall \& Stoica, 1994 & $\begin{array}{c}\text { Ratos Berlin Druckrey IV + } \\
\text { linhagem de adenocarcinoma } \\
\text { mamário ENU1564 }\end{array}$ & $\begin{array}{c}\text { redução da incidência e tamanho das } \\
\text { metástases esqueléticas }\end{array}$ \\
\hline Sasaki et al., 1995 & $\begin{array}{c}\text { Camundongos "nude" }+ \\
\text { linhagem de câncer de mama } \\
\text { MDA-MB-231 }\end{array}$ & $\begin{array}{l}\text { inibição do desenvolvimento de } \\
\text { metástases ósseas }\end{array}$ \\
\hline $\begin{array}{l}\text { Stearns \& Wang, } \\
1996\end{array}$ & $\begin{array}{c}\text { Camundongos SCID + } \\
\text { linhagem de câncer de próstata } \\
\text { PC-3 ML }\end{array}$ & $\begin{array}{l}\text { inibição do desenvolvimento de } \\
\text { metástases ósseas }\end{array}$ \\
\hline Yoneda et al., 1997 & $\begin{array}{c}\text { Camundongos "nude" }+ \\
\text { linhagem de câncer de mama } \\
\text { MDA-MB-231 }\end{array}$ & $\begin{array}{c}\text { redução ou inibição das lesões } \\
\text { osteolíticas }\end{array}$ \\
\hline Sasaki et al., 1998 & $\begin{array}{c}\text { Camundongos "nude" }+ \\
\text { linhagem de câncer de mama } \\
\text { MDA-MB-231 }\end{array}$ & $\begin{array}{l}\text { inibição do desenvolvimento de } \\
\text { metástases ósseas }\end{array}$ \\
\hline
\end{tabular}

Tabela 3. Trabalhos pré-clínicos sobre a ação dos bisfosfonatos em linhagens não-tumorais

\begin{tabular}{c|c|c}
\hline Autor & Linhagem & Propriedade \\
\hline Sahni et al., 1993 & osteoblastos & inibição da atividade dos osteoclastos \\
\hline Pennanen et al., 1995 & macrófagos & $\begin{array}{c}\text { inibição da secreção de citocinas e fatores de } \\
\text { crescimento }\end{array}$ \\
\hline Sansoni et al., 1995 & monócitos & inibição da função apresentadora de antígeno \\
\hline Wood et al., 2002 & $\begin{array}{c}\text { células endoteliais } \\
\text { humanas }\end{array}$ & inibição da angiogênese \\
\hline Fournier et al., 2002 & $\begin{array}{c}\text { células endoteliais } \\
\text { humanas }\end{array}$ & inibição da angiogênese \\
\hline
\end{tabular}

investigado o efeito anti-tumoral dos BFs YM 529 e YM 175 em linhagens celulares de vários tumores hematopoéticos, Resultados de estudos em células de câncer de mama humanas indicam que os BFs também possuem efeito anti-tumoral direto nestas células, uma vez que induzem apoptose em linhagens das mesmas. ${ }^{1,37,38}$ Esses trabalhos sugerem 0 envolvimento de caspases neste processo apoptótico, ${ }^{1,37}$ em especial da caspase $3 .^{38}$ Já foi demonstrado por Senaratne et al. que a expressão da proteína $\mathrm{Bcl}-2$ está diminuída em células da linhagem de câncer de mama M D A-M B-231 expostas ao pamidronato. ${ }^{1} \mathrm{Em}$ trabalho mais recente, 0 mesmo grupo demonstrou que a expressão induzida de $\mathrm{BCl}-2$ nesta mesma linhagem inibe eficientemente a fragmentação do DNA induzida pelo zoledronato, que é conseqüência da ativação das caspases em células de câncer de mama. ${ }^{38}$

Em pesquisa recente realizada por Senaratne et al. foi observado que o zoledronato impediu a localização na membrana da proteína Ras em linhagens de câncer 
de mama, indicando uma prenilação reduzida da mesma. ${ }^{38}$ Essas observações sugerem que 0 processo apoptótico mediado pelo zoledronato pode ser iniciado pela inibição de enzimas da via do mevalonato levando a um impedimento da prenilação de pequenas GT Pases. ${ }^{38}$

Trabal ho publicado por Jagdev et al. demonstrou uma ação sinérgica entre paclitaxel e zoledronato na redução do número de células de câncer de mama e indução de apoptose das mesmas. ${ }^{28} 0$ mecanismo pelo qual este efeito ocorre não foi determinado, mas a inibição da via do mevalonato pelo zoledronato e a prevenção da segregação cromossômica e da divisão celular pelo paclitaxel podem resultar em um efeito apoptótico cumulativo. ${ }^{28}$

0 utros trabalhos demonstram a inibição da proliferação e/ou indução de apoptose in vitro por BFs em linhagens de melanoma ${ }^{39}$ e osteossarcoma humanos. ${ }^{40}$

\section{Inibição da adesão e da invasividade celular}

Van der Pluijm et al. apresentaram evidências de que a adesão de células da linhagem de câncer de mama MDA-M B-231 à matriz extracelular óssea pode ser inibida pelo tratamento prévio de ossos corticais e trabeculares com BFs. ${ }^{41}$ Em contrapartida, Boissier et al. observaram que 0 tratamento prévio de células de câncer de mama (M D A-M B-231 eM CF-7) ede próstata (PC -3) com BFs inibiu a adesão a matrizes extracelulares ósseas mineralizadas ou não, demonstrando um efeito direto dos BFs nas células tumorais, independente da necessidade de contato das mesmas com um mineral coberto por BFs. ${ }^{42}$

0 mecanismo pelo qual os BFs aminados inibem a adesão de células tumorais à matriz óssea permanece desconhecido mas, provavelmente, a modulação de moléculas de adesão tais como caderinas, lamininas e integrinas está envolvida. ${ }^{6} \mathrm{~N} 0$ entanto, em pesquisa realizada por Boissier et al., apesar de os autores demonstrarem que os BFs modulam a adesão celular, as drogas não afetaram a expressão de integrinas na membrana das células tumorais. ${ }^{42}$

Boissier et al. observaram que os BFs inibiram a atividade proteolítica de várias metaloproteinases em linhagens de câncer de mama (M DA-M B-231) e de próstata (PmPC -3)..$^{43}$ Stearns demonstrou que 0 alendronato inibiu a secreção, estimulada por TGF-b1, da metaloproteinase M M P-2 em células de câncer de próstata ( $\mathrm{PC}-3 \mathrm{ML}$ ), o que levou a um bloqueio na degradação do colágeno-1 e a conseqüente redução da capacidade de invasão da célula tumoral. ${ }^{44}$

Virtanen et al. observaram que a inibição da invasividade de células de câncer de próstata (PC -3) induzida pelo alendronato era similar à induzida pela mevastatina (inibidor da via do mevalonato) e que ambas foram moduladas pelos intermediários geranilgeraniol e trans-trans-farnesol. ${ }^{45} 0$ pré-tratamento com alendronato também inibiu a migração celular. Os resultados deste trabalho demonstraram que a via do mevalonato, que leva à prenilação de proteínas, pode ser importante para a invasividade e migração de células tumorais in vitro. ${ }^{45}$

\section{Inibição de metástases ósseas in vivo}

Através da inibição da reabsorção óssea mediada por osteoclasto, os BFs diminuem a liberação pelo osso de fatores de crescimento promotores de tumor e retardam a progressão de metástases ósseas. Como resultado, não só a osteólise é reduzida, mas também ocorreuma redução no número eno tamanho detumores no 0sso. ${ }^{6}$

Sasaki et al. ${ }^{46}$ e $\mathrm{H}$ all e Stoica ${ }^{47}$ examinaram o efeito do risedronato em modelos animais de metástases ósseas e observaram que a droga reduziu a incidência, ${ }^{47}$ diminuiu a progressão ou inibiu o desenvolvimento ${ }^{46} \mathrm{e}$ diminuiu o volume tumoral de metástases ósseas. ${ }^{46,47}$

Vários outros estudos também demonstraram a inibição de metástases ósseas por diversos BFs em modelos animais. ${ }^{48,49,50,51,52}$ Em contrapartida, Shevrin et al. não observaram redução na incidência, tamanho ou número de metástases ósseas com o uso de etidronato em um modelo de metástase usando a linhagem celular de câncer de próstata humano PC $-3 .^{53}$

Estudo recente publicado por $M$ ichigami et al. demonstrando os efeitos do ibandronato em modelos de metástases em camundongos, sugere que os BFs não são efetivos em metástases viscerais quando administrados tanto preventivamente quanto terapeuticamente. ${ }^{54}$

$0 \mathrm{~s}$ resultados de estudos randomizados em pacientes com câncer de mama primário que receberam oralmente $1600 \mathrm{mg} /$ dia de clodronato são conflitantes. Um dos trabalhos, ${ }^{55}$ realizado com 302 pacientes, mostrou uma redução da incidência e do número de novas metástases ósseas e viscerais e um aumento da sobrevida. O utro estudo, ${ }^{56}$ compreendendo 299 pacientes, não encontrou nenhum efeito nas metástases ósseas, mas um aumento no número de metástases viscerais e uma redução na sobrevida. Resultados de um estudo controlado mais recente, ${ }^{57}$ compreendendo 1.069 pacientes, mostraram uma redução significativa na ocorrência de metástases ósseas apenas durante o período de tratamento e redução na mortalidade, porém nenhum efeito nas metástases viscerais. 0 utros estudos randomizados foram iniciados com clodronato e outros BFs, incluindo pamidronato e 
zoledronato intravenosos eibandronato oral. ${ }^{58}$

\section{Inibição da angiogênese}

Foi demonstrado em trabalho recente que 0 zoledronato é um potente inibidor da angiogênese. ${ }^{59}$ In vitro esta droga inibiu a proliferação de células endoteliais (CE) humanas estimuladas com soro fetal bovino, fator de crescimento de fibroblasto básico (bFGF) e fator de crescimento endotelial vascular (VEGF). Em anéis aórticos cultivados e em ensaios com membrana corioalantóica de ovo de galinha, 0 zoledronato reduziu o brotamento de vasos. Q uando administrado sistemicamente a camundongos 0 zoledronato inibiu potentemente a angiogênese induzida por implantes subcutâneos impregnados com bFG F. ${ }^{59}$

Fournier et al. observaram que 0 tratamento de CE com BFs reduziu a proliferação, induziu apoptose e diminuiu a formação de tubos similares a capilares in vitro. ${ }^{60}$ Este grupo também estudou os efeitos dos BFs na revascularização da próstata induzida por testosterona em ratos castrados, visto que foi anteriormente observado que os BFs acumulam-se transitoriamente na próstata desses animais, e observaram que tanto o IBA quanto o ZOL induziram redução de $50 \%$ da revascularização da próstata. ${ }^{60} \mathrm{~N}$ este mesmo trabalho, os autores quantificaram vasos sangüíneos em biópsias ósseas de pacientes com doença de Paget antes e após tratamento com clodronato e constataram uma redução de $40 \%$ da vascularização após o tratamento com o BF.

Em estudo realizado por Santini et al. em 25 pacientes com metástases ósseas e tratados com pamidronato, observou-se uma diminuição significativa e persistente dos níveis séricos de VEGF, sugerindo um efeito antiangiogênico desta droga. ${ }^{61}$

\section{Efeitos na secreção de citocinas e fatores de crescimento}

No complexo micro-ambiente de uma metástase óssea, altas concentrações locais de BFs podem exercer efeitos não apenas nos osteoclastos e nas células tumorais, mas também em outros tipos celulares que influenciam a proliferação de células tumorais através da liberação de fatores, como osteoblastos, células estromais da medula óssea e monócitos. ${ }^{6}$

Pennanen et al.(1995) demonstraram que os BFs possuem efeito inibitório na secreção de citocinas (IL1, I L-6 e T N Fa) por macrófagos da linhagem RAW 264 in vitro. ${ }^{62}$

Sahni et al. sugerem que parte da inibição da atividade dos osteoclastos pelos BFs seria mediada pela ação de osteoblastos, pois a diminuição do agente estimulatório encontrado no meio condicionado por estas células inibiria a atividade reabsortiva daquelas ${ }^{63}$

Sansoni et al. observaram que 0 alendronato inibe a função apresentadora de antígeno dos monócitos provavelmente através da inibição da produção de IL-1, o que poderia contribuir para o efeito inibitório da droga na reabsorção óssea. ${ }^{64}$

\section{CONCLUSÕES E PERSPECTIVAS}

O s resultados de uma série de estudos recentes revelam que os BFs possuem efeito anti-tumoral em diferentes tipos de neoplasias e que o mecanismo de ação destas drogas também pode ser diverso, incluindo inibição da proliferação celular, indução de apoptose, inibição da adesão e da invasividade celular, inibição da angiogênese e efeitos na secreção de fatores de crescimento e citocinas pelo micro-ambiente tumoral.

Um entendimento mais profundo e completo sobre os mecanismos pelos quais os BFs influenciam 0 potencial metastático ou induzem a apoptose podem sugerir possibilidades terapêuticas novas e seletivas.

\section{REFERÊNCIAS BIBLIOGRÁFICAS}

1. SenaratneSG, Pirianov G, M ansi J L, ArnettT R, Colston $\mathrm{KW}$. Bisphosphonates induce apoptosis in human breast cancer cell lines. Br J C ancer 2000;82:1459-68.

2. Fleisch H. Diphosphonates: history and mechanisms of action. M etab BoneD is Rel Res 1981;4/5:279-88.

3. Fleisch $\mathrm{H}$. Bisphosphonates. Pharmacology and use in the treatment of tumour-induced hypercal caemic and metastatic bone disease. D rugs 1991;42:919-44.

4. Berruti A, D ogliotti L, Tucci M , Tarabuzzi R, Fontana D, Angeli A. M etabolic bonediseaseinduced by prostate cancer: rationale for the use of bisphosphonates. J U rol 2001;166:2023-31.

5. Brown D L, RobbinsR. D evelopmentsin thetherapeutic applications of bisphosphonates. J Clin Pharmacol 1999;39:651-60.

6. G reen JR. Anti-tumor potential of biphosphonates. M ed Klin 2000;95 Suppl 2:23-8.

7. Boonecamp PM, van der WeePals LJA, van Wijk-van Lennep M M L, Thesing CW, Bijvoet O L. Two modes of action of bisphosphonates on osteoclastic resorption of mineralized matrix. J BoneM iner Res 1986;1:27-39.

8. Ryzen $E, M$ artodan $R$, Troxell M , Benson A, Paterson A, Shepard $K$, et al. Intravenous etidronate in the management of malignant hypercalcaemia. Arch Intern M ed 1985; 145:449-52.

9. KanisJA, U rwin GH, Gray RE, Beneton M N , M cCloskey $\mathrm{EV}, \mathrm{H}$ amdy $\mathrm{N} A$, et al. Effects of intravenous etidronate on skeletal and calcium metabolism. Am J M ed 1987;82:55-70. 
10. Berenson J R, Linchtenstein A, Porter L, D imopoulos M A, Bordoni R, George $S$, et al. Efficacy of pamidronate in reducing skeletal events in patients with advanced multiple mieloma. M yeloma Aredia Study Group. N Engl J Med 1996;334:488-93.

11. H ortobagyi GN , Theriault RL, Porter L, Blayney D, Lipton $A$, Sinoff $C$, et al. Efficacy of pamidronate in reducing skeletal complications in patients with breast cancer and lytic metastasis. Protocol 19. A redia B reast C ancer Study Group. N Engl J M ed 1996;335:1785-91.

12. ErnstDS, $M$ acD onald RN , Paterson AH , Jensen J, Brasher $P, B$ ruera E. A doubleblind cross-over trial of intravenous clodronatein metastatic bonepain. J Pain Symptom M anage 1992;7:4-11.

13. Purohit O P, Anthony C, Radstone CR, $O$ wen J , C oleman RE. H igh-doseintravenous pamidronatefor metastatic bone pain. Br J Cancer 1994;70:554-8.

14. H ughes DE, M acD onald $B R$, R ussell RG G, G owen M . Inhibition of osteoclast-like cell formation by bisphosphonates in long-term cultures of human bone marrow. J Clin Invest 1989;83:1930-5.

15. Hughes DE, Wright $K R$, Uy $H L$, Sasaki $A$, Yoneda $T$, Roodman GD, et al. Bisphosphonates promote apoptosis in murineosteoclastsin vitro and in vivo.J BoneM iner Res 1995;10:1478-87.

16. Selander KS, M onkkonen J, Karhukorp E, $H$ arkonen $P$, $H$ annuniemi $R$, Vaananen KK. Characteristics of clodronate induced apoptosisin osteoclasts and macrophages. M ol Pharmacol 1996;50:1127-38.

17. Rodan GA. M echanisms of action of bisphosphonates. Annu Rev Pharmacol Toxicol 1998;38:375-88.

18. 0 wens J M , Fuller K, Chambers T J 0 steoclast activation: potent inhibition by the bisphosphonate alendronate through a nonresorptive mechanism. J Cell Physiol 1997;172:79-86.

19. Plasmans C M T, Jap PH K, KuypersW, Slooff TJJ. Influence of a diphosphonate on the cellular aspect of young bonetissue. CalcifTissuelnt 1980;32:247-56.

20. Sato M , G rasser W, Endo N, Akins R, Simmons H , Thompson D D, et al. Bisphosphonates action. Alendronate localization in rat boneand effects on osteoclast ultrastructure. J Clin Invest 1991;88:2095-105.

21. NesbittSA, H orton M A. Trafficking of matrix collagensthrough boneresorbing osteoclasts. Science 1997;276:266-69.

22. Salo J, Lenenkari P, M ulari M , M etsikko K, Vaananen H K. Removal of osteoclast bone resorption products by transcytosis. Science 1997;276:270-73.

23. Frith JC, M onkkonen J, Blackburn G M , Russell RGG, Rogers M. Clodronate and liposome-encapsulated clodronate aremetabolized to atoxic AT $P$ analog, adenosine 5'-(b,g-dichloromethylene) triphosphate, by mammalian cells in vitro.J BoneM iner Res 1997;12:1358-67.

24. Luckman SP, C oxon FP, Ebetino FH , Russell RG G, Rogers
M J. H eterocycle-containing bisphosphonates cause apoptosis and inhibit bone resorption by preventing protein prenylation: evidencefrom structure activity relationships in 1774 macrophages. J Bone Miner Res 1998;13:1668-78.

25. Benford H L, Frith JC, AuriolaS, M önkkönen J, Rogers] . Farnesol and geranylgeraniol prevent activation of caspases by aminobisphosphonates: biochemical evidence for two distinct pharmacological classes of bisphosphonatedrugs. M ol Pharmacol 1999;56:131-40.

26. Fisher JE, Rogers M J, H alasy JM , Luckman P, H ughesDE, $M$ asarachia PJ, et al. Alendronate mechanism of action: geranylgeraniol, an intermediatein the mevalonate pathway, preventsinhibition of osteoclast formation, boneresorption, and kinaseactivation in vitro. Proc $\mathrm{N}$ atl Acad Sci USA 1999;96:133-8.

27. ReszkaAA, H alasy-N agy JM , M asarachia PJ, Rodan GA. Bisphosphonates act directly on the osteoclast to induce caspase cleavage of mstl kinase during apoptosis. J Biol Chem 1999;274:34967-73.

28. Jagdev SP, Coleman RE, Shipman CM, Rostami HA, Croucher PI. Thebisphosphonate, zoledronic acid, induces apoptosis of breast cancer cells: evidence for synergy with paclitaxel. Br J Cancer 2001;84:1126-34.

29. Coxon FP, Benford HL, Russel RG G, Rogers M J. Protein synthesisis required for caspaseactivation and induction of apoptosis by bisphosphonate drugs. M ol Pharmacol 1998;54:631-8.

30. Benford H L, M CG owan N W, H elfrich M H , N uttal ME, Rogers MJ. Visualization of bisphosphonateinduced caspase 3 activity in apoptotic osteoclasts in vitro. Bone 2001;28:465-73.

31. Shipman CM , Rojers MJ, Apperley JF, Russell RGG, Croucher $\mathrm{PI}$. Bisphosphonatesinduceapoptosisin human myeloma cell lines: a novel anti-tumour activity. $\mathrm{Br}$ J $\mathrm{H}$ aematol 1997;98:665-72.

32. Aparicio A, Gardner A, Tu Y, Savage A, Berenson J, Lichtenstein $A$. In vitro cytoreductive effects on multiple myeloma cells induced by bisphosphonates. Leukemia 1998;12:220-9.

33. Shipman CM , C roucher PI, Russell RG G, H elfrich M H , Rojers M J. Thebisphosphonates incadronate (YM 175) causes apoptosis of human myel oma cells in vitro by inhibiting the mavalonate pathway. Cancer Res 1998;58:5294-7.

34. G ordon S, H elfrich M H, Sati H I, G reaves M , Ralston SH, Culligan DJ, et al. Pamidronatecausesapoptosis of plasma cells in vivo in patients with multiple myeloma. $\mathrm{Br}$ J H aematol 2002;119:475-83.

35. Tassone P, Forciniti S, Galea E, M orrone G, Turco M C, $M$ artinelli V, et al. Growth inhibition and synergistic induction of apoptosis by zoledronate and dexamethasone in human myeloma cell lines. Leukemia 2000;14:841-4. 
36. Nishida S, Kikuichi S, H aga H, Yoshioka S, Tsubaki M, Fujii $\mathrm{K}$, et al. Apoptosis-inducing effect of a new bisphosphonate, YM 529, on various hematopoietic tumor cell lines. Biol Pharm Bull 2003;26:96-100.

37. Fromigue 0 , Lagneaux L, Body JJ. Bisphosphonates induce breast cancer cell death in vitro. J Bone M iner Res 2000;15:2211-21.

38. SenaratneSG, M ansi J L, Colston KW. Thebisphosphonate zoledronic acid impairs membranelocalisation and induces cytochrome c release in breast cancer cells. Br J Cancer 2002;86:1479-86.

39. Riebeling C, Forsea AM , Raisova M , O rfanos CE, G eilen CC. The bisphosphonatepamidronateinduces apoptosis in human melanoma cells in vitro. $\mathrm{Br} J \mathrm{C}$ Cancer 2002;87:366-71.

40. Sonnemann J, Eckervogt V, Truckenbrod B, Boos J, W inkelmann W, van Valen F. The bisphosphonate pamidronateis a potent inhibitor of human osteosarcoma cell growth in vitro. Anticancer D rugs 2001;12:459-65.

41. Van der Pluijm $G$, Vloedgraven $H$, van Beek $E$, van der WeePalsL, Löwik C, PapapoulosS. Bisphosphonatesinhibit theadhesion of breast cancer cells to bonematricesin vitro. J Clin Invest 1996;98:698-705.

42. Boissier S, M agnetto S, Frappart L, C uzin B, Ebetino FH , D elmas PD, et al. Bisphosphonates inhibit prostate and breast carcinoma cell adhesion to unmineralized and mineralized bone extracellular matrices. Cancer Res 1997;57:3890-4.

43. Boissier $S$, Ferreras $M$, Peyruchaud $O, M$ agnetto $S$, Ebetino $\mathrm{FH}, \mathrm{C}$ olombel $\mathrm{M}$, et al. Bisphosphonatesinhibit breast and prostatecarcinomacell invasion, an early event in theformation of bonemetastases. C ancer Res 2000;60:2949-54.

44. Stearns M E. Alendronateblocks T G F-b1 stimulated collagen 1 degradation by human prostate PC -3 M L cells. Clin Exp M etastasis 1998;16:332-9.

45. Virtanen SS, Väänänen H K, H ärkönen PL, Lakkakorpi PT. Alendronateinhibits invasion of $\mathrm{PC}-3$ prostatecancer cells by affecting the mevalonate pathway. Cancer Res 2002;62:2708-14.

46. Sasaki A, Boyce BF, Story B, Wright KR, Chapman M , Boyce $R$, et al. Bisphosphonate risedronate reduces metastatic human breast cancer burden in bonein nude mice. Cancer Res 1995;55:3551-7.

47. H all D G, Stoica G. Effect of thebisphosphonaterisedronate on bone metastases in a rat mammary adenocarcinoma model system. J BoneM iner Res 1994;9:221-30.

48. W ingen F, Eichmann T, M anegold C, K rempien B. Effects of new bisphosphonic acids on tumor-induced bone destruction in therat. J Cancer ResClin O ncol 1986;111:35-41.

49. Krempien B, W ingen F, Eichmann T, M uller M, Schmahl $D$. Protective effects of a prophylactic treatment with the bisphosphonate 3-amino-1-hydroxypropane-1,1bisphosphonic acid on the development of tumor osteopa- thies in the rat: experimental studies with theWalker carcinosarcoma 256. O ncology 1988;45:41-6.

50. Stearns M E, Wang M . Effects of alendronateand taxol on PC -3 M L cell bone metastases in SCID mice. Invasion M etastasis 1996;16:116-31.

51. YonedaT, Sasaki A, D unstan C, Williams PJ, Bauss F, D e Clerck YA, et al. Inhibition of osteolytic bonemetastasis of breast cancer by combined treatment with the bisphosphonateibandronateand tissueinhibitor of thematrix metalloproteinase-2.J Clin Invest 1997;99:2509-17.

52. Sasaki A, Kitamura K, Alcalde RE, Tanaka T, Suzuki A, Etoh $Y$, et al. Effect of a newly devel oped bisphosphonate, YH 529, on osteolytic bonemetastases in nude mice. Int J Cancer 1998;77:279-85.

53. Shevrin D H , G orny KI, Rosol TJ, Kukreja SC. Effect of etidronato disodium on the development of bonelesions in an animal model of bone metastasis using the human prostatecancer cell linePC -3. Prostate 1991;19:149-54.

54. M ichigami T, H iraga T, W illiams PJ, N iewolna $M$, $N$ ishimura $R, M$ undy $G R$, et al. The effect of the bisphosphonateibandronate on breast cancer metastasisto visceral organs. Breast $C$ ancer R esTreat 2002;75:249-58.

55. Diel IJ, Solomayer EF, C osta SD , G ollan G, G oerner R, Wallwiener $D$, et al. Reduction in new metastases in breast cancer with adjuvant clodronatetreatment. N Engl J M ed 1998;339:357-63.

56. Saarto T, Blomqvist C, Virkkunen P, Elomaa I. Adjuvant clodronatetreatment doesnot reducethefrequency of skeletal metastasesin node-positive breast cancer patients: 5year results of a randomized controlled trial. J Clin 0 ncol 2001;19:10-7.

57. Powles T, Paterson S, Kanis JA, M cCloskey E, A shley S, Tidy A, et al. Randomized, placebo-controlled trial of clodronatein patientswith primary operablebreast cancer. J Clin O ncol 2002;20:3219-24.

58. Paterson AH . Adjuvant bisphosphonate therapy: the future. Semin 0 ncol 2001;28(4 Suppl 11):81-5.

59. WoodJ, Bonjean K, RuezzS, BellahceneA, D evy L, Foidart $J M$, et al. Novel antiangiogenic effects of the bisphosphonate compound zoledronic acid.J Pharmacol Exp Ther 2002;302:1055-61.

60. Fournier P, Boissier S, Filleur S, Guglielmi J, Cabon F, Colombel $M$, et al. Bisphosphonatesinhibit angiogenesis in vitro and testosterone-stimulated vascular regrowth in the ventral prostate in castrated rats. Cancer Res 2002;62:6538-44.

61. Santini D, Vicenzi B, Avisati G, Dicuonzo G, Battistoni F, $G$ avasci $M$, et al. Pamidronate induces modifications of circulating angiogenetic factors in cancer patients. $\mathrm{Clin}$ Cancer Res 2002;8:1080-4.

62. Pennanen N , Lapinjoki S, U rtti A, M onkonen J. Effect of liposomal and free bisphosphonates on the I L-1 beta, I L- 6 and TNF alpha secretion from RAW 164 cells in vitro. 
Pharm Res 1995;12:916-22.

63. Sahni M, Guenther H L, Fleisch H, Collin P, M artin TJ. Bisphosphonates act on rat bone resorption through the mediation of osteoblasts. J C lin Invest 1993;91:2004-11.
64. Sansoni P, Passeri G, Fagnoni F, M ohagheghpour N, Snelli $G$, Brianti V, et al. Inhibition of antigen-presenting cell function by alendronate in vitro. J Bone Miner Res 1995;10:1719-25. 